\title{
Development of a novel capacitance electrochemical biosensor based on silicon nitride
} for ochratoxin A detection

Madiha Bougrini ${ }^{\mathrm{a}, \mathrm{de}}$, Abdoullatif Baraket ${ }^{\mathrm{a}}$, Talha Jamshaid ${ }^{\mathrm{b}}$, Abdelhamid El Aissari ${ }^{\mathrm{b}}$, Joan Bausells ${ }^{\mathrm{c}}$, Miguel Zabala $^{\mathrm{c}}$, Nezha El Bari ${ }^{\mathrm{d}}$, Benachir Bouchikhi ${ }^{\mathrm{e}}$, Nicole Jaffrezic-Renault ${ }^{\mathrm{a}}$, Errachid Abdelhamid ${ }^{\mathrm{a}}{ }^{*}$, Nadia Zine $^{\mathrm{a}}$

\footnotetext{
${ }^{a}$ Université Claude Bernard Lyon1, UMR 5280, Institut des Sciences Analytiques, 5, rue de la Doua, 69100 Villeurbanne, France

b Université Claude Bernard Lyon1, LAGEP-CPE, 43 Bd. 11 Nov. 1918, F-69622 Villeurbanne, France

${ }^{\mathrm{c}}$ Institut de Microelectronica de Barcelona, IMB-CNM(CSIC), Campus UAB, 08193 Bellaterra, Barcelona, Spain

d Biotechnology Agroalimentary and Biomedical Analysis Group, Department of Biology, Faculty of Sciences, Moulay Ismaïl University, B.P. 11201,Zitoune, 50003 Meknes, Morocco

${ }^{\text {e }}$ Sensor Electronic \& Instrumentation Group, Department of Physics, Faculty of Sciences, Moulay Ismail University, B.P. 11201, Zitoune, Meknes, Morocco
}

\begin{abstract}
We report in this paper about the development of a novel capacitance electrochemical biosensor based on silicon nitride substrate $\left(\mathrm{Si}_{3} \mathrm{~N}_{4}\right)$ combined with a new structure of magnetic nanoparticles (MNPs). $\mathrm{Si}_{3} \mathrm{~N}_{4}$ is highly stable as it was fabricated by a combination of several layers of Aluminum (Al), silicon p-doped (Sip), silicon dioxide $\left(\mathrm{SiO}_{2}\right)$ and silicon nitride $\left(\mathrm{Si}_{3} \mathrm{~N}_{4}\right)$. This structure $\left(\mathrm{Si}_{3} \mathrm{~N}_{4} / \mathrm{SiO}_{2} / \mathrm{Si}-\mathrm{p} / \mathrm{Al}\right)$ has provided several advantages compared with other materials commonly used, and in particular in solid state physics for electronic-based biosensors. The MNPs with terminated carboxylic acid were covalently bonded to $\mathrm{Si}_{3} \mathrm{~N}_{4}$ through a Self-Assembled Monolayers (SAMs) of the silane-amine (3-Aminopropyl) triethoxysilane (APTES). Finally anti-ochratoxin A antibodies were immobilized on MNPs by amide bonding. Contact Angle measurements, Atomic Force Microscopy, Scanning Electron Microscopy and Fluorescence Microscopy characterizations were performed during the biofunctionalization of the biosensor surface. Electrochemical measurements were carried out using Mott-Schottky analysis for ochratoxin A detection. The biosensor was highly sensitive and specific for ochratoxin A antigens, with a limit of detection of $4.57 \mathrm{pM}$, when compared to other interferences ochratoxin B and aflatoxin G1. The measurements were highly stable and reproducible for detection and interferences. The proposed method is very promising for ochratoxin A detection of several agro-food industry applications.
\end{abstract}

\section{Keywords:}

Capacitive biosensor, Silicon nitride, Magnetic nanoparticles, Mott-Schottky analysis, Ochratoxin A

Corresponding author: abdelhamid.errachid@univ-lyon1.fr (E. Abdelhamid).

http://dx.doi.org/10.1016/j.snb.2016.03.166 


\section{Introduction}

Ochratoxin A (OTA), is a secondary fungal metabolite produced by various Aspergillus and Penicillium strains, which was found to be one of the predominant contaminating mycotoxins in a wide variety of food commodities such as cereals, dried fruits, nut, spices, coffee beans, cocoa, beer, wine, etc [1-3]. In the European Union, some regulatory limits have already been introduced for the levels of OTA in food products such as raw cereal grains $(5 \mathrm{~g} / \mathrm{kg})$, dried fruits $(10 \mathrm{~g} / \mathrm{kg})$, roasted coffee and coffee products $(5 \mathrm{~g} / \mathrm{kg})$, grape juice ( $2 \mathrm{~g} / \mathrm{kg}$ ) (EC No. 123/2005) and also for all types of wines $(2 \mathrm{~g} / \mathrm{kg})$ [4]. Currently, the methods commonly used for OTA detection are based on high-performance liquid chromatography (HPLC) with fluorescent detection [5]; theLOD of the proposed method was $0.0025 \mathrm{~g} / \mathrm{L}$. However, HPLC is laborious, time-consuming, and requires sophisticated equipment and qualified personnel. Furthermore, gas chromatography coupled with mass spectrometry (GC-MS) [6] or enzyme-linked immunosorbent assay (ELISA) [7] have also been used for OTA detection and the LOD was around $0.15 \mathrm{ng} / \mathrm{mL}$. Although this technique is used for rapid screening and allows multiple analyses in a short time, it remains limited by usinglabeled bioreagents which are expensive. Toovercome the abovelimitations, several types of biosensors have aroused the interest of researchers forOTAdetection.

In the last years, several optical and electrochemical techniques based on biosensors have been investigated for OTA detection [8-10]. Liu et al. have reported about an ultrasensitive electrochemical immunosensor for ochratoxin A using gold colloid-mediated hapten immobilization. The detection limit achieved in this work was $8.2 \mathrm{pg} / \mathrm{mL}$ [9]. Biosensors used for this interest in the literature were mainly based on gold or polymer substrate combined with SAMs or cross-linkers for biomolecules immobilization [11]. The functionalization of the biosensor surface with SAMs is the most important step for the development of robust and stable biosensors. Recently, Silicon nitride substrate $\left(\mathrm{Si}_{3} \mathrm{~N}_{4}\right)$ based microand nano-fabrication technology has been successfully combined with biochemistry, enabling the fabrication of novel biosensing devices with high sensitivity and selectivity [12-14]. This material offers more advantages when compared to others, such as the absence of undesirable impurities and the excellent control of the film composition and thickness. This is especially important for ultra-thin layers used in gate and tunnel dielectrics in the fabrication of biosensor devices with metal-oxide-semiconductor (MOS) technology [15]. The combination of its electronic and mechanical properties $[13,14,16]$ makes $\mathrm{Si}_{3} \mathrm{~N}_{4}$ an extremely attractive material for biosensor applications. There is a large work in the literature based on SAMs on different metals [17-20], in particular thiols on gold. However, less work has been devoted to the study of the electronic properties of SAMs deposited on $\mathrm{Si}_{3} \mathrm{~N}_{4}$. The surface of the latter is usually modified with organosilanes carrying chemically active groups [21,22], to provide a suitable interface between silicon-based transducer and immobilized biomolecules.

Beside the choice of a good and stable material for biosensor substrates, other spherical nano particles (NPs) have been used as immobilization support in biosensing technology in order to enhance the sensitivity of the biosensors [23]. These NPs are available with a wide variety of surface functional groups and show advantages in the process of immobilization by increasing the surface area, the stability of the surface-bound antibodies, improving orientation of the immobilized antibody as well as achieving faster assay kinetics [24-26].

The aim of the present work was to develop a sensitive, selective, cost-effective, and comparatively fast method for quantitative OTA detection. The silicon nitride substrate $\left(\mathrm{Si}_{3} \mathrm{~N}_{4}\right)$ combined with a new structure of mangnetic nanoparticles (MNPs) was used to develop an electrochemical biosensor for OTA detection. Magnetic Nanoparticles (MNP) were composed from a conductive core and carboxylic acid modified shell allowing amid bonding with OTA antibodies. This electrochemical structure using Mott-Schottky analysis was proposed in our knowledge for the first time for OTA detection. In order to control all steps of the biosensing layer, the surface of the biosensor has been characterized by Contact Angle (CA) measurements, Atomic Force Microscopy (AFM), Scanning Electron Microscopy (SEM), and fluorescence microscopy. 


\section{Experimental}

\subsection{Chemicals and reagents}

Ochratoxin A (OTA), ochratoxin B (OTB), aflatoxin G1 (AFG1), potassium chloride ( $\mathrm{KCl}$ ), hydrogen chloride $(\mathrm{HCl})$, sodium hydroxide $(\mathrm{NaOH})$, sulfuric acid $(98 \%)\left(\mathrm{H}_{2} \mathrm{SO}_{4}\right)$, ethanol $(98 \%)$, hydrogen peroxide $(30 \%)$ $\left(\mathrm{H}_{2} \mathrm{O}_{2}\right)$, N-hydroxysuccinimide (NHS), 1-ethyl3-(3-dimethylaminopropyl)-carbodiimide hydrochloride (EDC), (3-Aminopropyl) triethoxysilane (APTES), ethanolamine (ETA), octadecyltrichlorosilane (OTS), styrene (St), divinyl-benzene (DVB), 4,4'-Azobis(4-cyanopentanoic acid) (ACPA) and sodium dodecyl sulfate solution (SDS) were all supplied by Sigma-Aldrich. The monoclonal anti-OTA antibody was purchased from Abcam. Polydimethylsiloxane (PDMS) was purchased from Dow Corning, France. Phosphate buffer saline (PBS) with pH 7.4 was used in MottSchottky experiments.

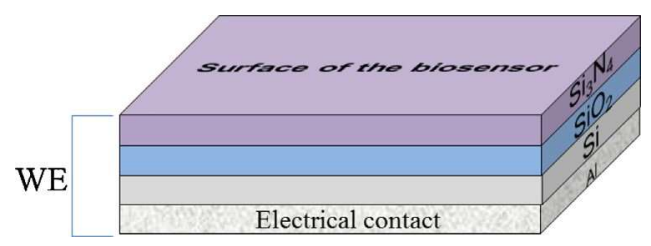

Fig. 1. Schematic illustration of the capacitive biosensor based on $\mathrm{Si}_{3} \mathrm{~N}_{4} / \mathrm{SiO}_{2} / \mathrm{Si} / \mathrm{Al}$ substrate.

\subsection{Process for $\mathrm{Si}_{3} \mathrm{~N}_{4}$ Substrate fabrication}

$\mathrm{Si}_{3} \mathrm{~N}_{4}$ coated Si substrates $<100>\left(\mathrm{Si}-\mathrm{p} / \mathrm{SiO}_{2} / \mathrm{Si}_{3} \mathrm{~N}_{4}\right) 1.2 \mathrm{~cm} \times 1.2 \mathrm{~cm}$ in size were obtained from the Centre Nacional de Microelectrònica (CNM-IMB, CSIC, Spain). Silicon wafers were thermally oxidized at $850{ }^{\circ} \mathrm{C}$ (78 nm thickness) followed by a deposition of a thin layer $\left(100 \mathrm{~nm}\right.$ ) of $\mathrm{Si}_{3} \mathrm{~N}_{4}$ using low-pressure chemical vapor deposition. The Si-p layer was uniformly doped with boron (dose $\left.1 \times 10^{15} \mathrm{~cm}^{-2}\right)$. An ohmic contact was realized by a deposition of $1 \mu \mathrm{m}$ of aluminum on the backside of the wafer (Fig. 1).

\subsection{Magnetic nanoparticle preparation}

Core-shell structure was prepared using a given St/DVB weight ratio (20 wt.\% St and 80 wt.\% DVB), 2 wt.\% ACPA was used as an initiator and solubilized first in $0.1 \mathrm{M} \mathrm{NaOH}$ solution. Total amount $(1200 \mathrm{~L})$ of St/DVB respectively was used. The polymerization was carried out in a $60 \mathrm{~mL}$ three necked double wall glass reactor made up of glass anchor type stirrer, a reflux condenser and a nitrogen inlet. The temperature was regulated at $70{ }^{\circ} \mathrm{C}$ by using a thermal bath. The SDS solution was prepared at a concentration below the critical micelle concentration (CMC) $1 \mathrm{gL}^{-1}$. Magnetic emulsion was finally dispersed in the SDS solution of the boiled and degassed Milli-Q water. First, $50 \mathrm{~mL}$ of magnetic emulsion was stabilized with an aqueous solution of anionic surfactant SDS via serum replacement process performing only one time separation/dispersion cycles and introduced in the reactor and purged under nitrogen for $1 \mathrm{~h}$ under stirring $(300 \mathrm{rpm})$ for removal of oxygen flux. After that introduced St and DVB at once into reactor for $70 \mathrm{~min}$ for swelling of magnetic droplets by monomers, temperature was rapidly increased to $70{ }^{\circ} \mathrm{C}$ and $2 \mathrm{wt} . \%$ ACPA (with respect to the weight of monomer and cross-linker) was added in the monomer swollen magnetic emulsion. The polymerization was run at constant stirring $(300 \mathrm{rpm})$ for $20 \mathrm{~h}$ till complete synthesis of magnetic latexes particles which having carboxylic group on surface as shown in Fig. S1 (Supplementary material).

\subsection{Contact angles measurements (CAM)}

Contact angle measurements were carried out using an Easy drop OCA 20, DataPhysics Instruments (Germany) to characterize silicon nitride substrate after each chemical surface modification. The measurements were analyzed with a droplet of $3 \mathrm{~L}$ of deionized water. Four values of CAM were recorded for each substrate. 


\subsection{Microcontact printing $(C P)$}

Micro-contact printing (CP) technique was used to confirm the MNPs bonding onto modified silicon nitride surface. For this interest, an elastomeric stamp based on PDMS was fabricated by replica molding (RM). Here, a mixture of pre-polymer PDMS and curing agent $(10: 1 \mathrm{w} / \mathrm{w})$ was poured onto a silanised silicon mold which contains micropillars on relief of its surface (Fig. 2a, b). The whole PDMS/silicon-mold degassed to ensure all

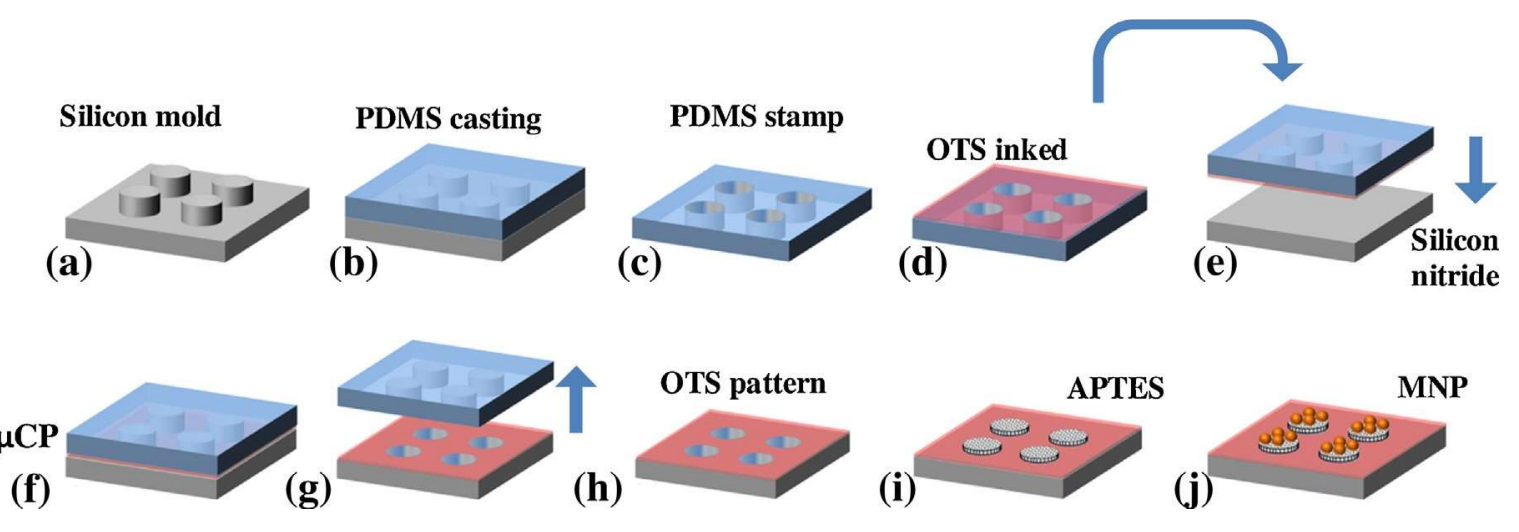

Fig. 2. Micro contact printing (CP) process to immobilize MNP onto APTES pattern.

air bubbles in the PDMS mixture were removed. This prevents the creation of defects on the PDMS stamp surface. After polymerization, the PDMS stamp was peeled-off from the silicon mold bearing the micro-holes on its surface (Fig. 2c). The stamp was then inked by immersion in heptane solution containing OTS $(5 \mu \mathrm{M})$ and carbon tetrachloride $(4 \mathrm{mM})$ for $1 \mathrm{~min}$ and dried with stream of nitrogen (Fig. 2d). The stamp was then brought into immediate and conformal contact with silicon nitride substrate previously activated by piranha (Fig. 2e, f). After $\mu$ CP, the PDMS stamp was peeled-off from silicon nitride surface (Fig. 2g). Here SAMs of OTS were formed on silicon nitride surface which was placed afterward on the oven for $45 \mathrm{~min}$ in order to enhance OTS adhesion on the surface (Fig. 2h). Silicon nitride subtract was immersed afterward in ethanol solution containing $1 \%$ of APTES for $30 \mathrm{~min}$, rinsed with ethanol, dried with a stream of nitrogen and placed again on the oven for $45 \mathrm{~min}$. The APTES silane was bonded onto the remaining active silicon nitride surface (Fig. 2i). Finally, the silicon nitride surface was dropped in $500 \mu \mathrm{L}$ of MNPs solution mixed with $0.4 \mathrm{M}$ EDC and $0.1 \mathrm{M}$ NHS for $3 \mathrm{~h}$ at $37{ }^{\circ} \mathrm{C}$. This allows a covalent bonding of MNPs with amine of APTES (Fig. 2j).

\subsection{Fluorescence microscopy}

Fluorescence images were taken using a fluorescence microscope (Zeiss Axioplan 2 Imaging apparatus, equipped with $10 \times$ and $40 \times$ lenses and a monochrome camera). Samples were observed by fluorescent light: OTA sample was excited with a $550( \pm 25) \mathrm{nm}$ band-pass filter and fluorescence from the sample was observed with a $605( \pm 70) \mathrm{nm}$ band-pass filter.

\subsection{Atomic force microscopy (AFM)}

Atomic Force Microscopy (AFM) was performed in air under ambient conditions using a Nano observer, CSI Company (France). AFM Nano-Observer has XY scan range $110 \mu \mathrm{M}$ (tolerance $\pm 10 \%$ ), $\mathrm{Z}$ range $9 \mu \mathrm{m}$ (tolerance $\pm 10 \%$ ) and $\mathrm{XY}$ drive resolution 24 bit control $-0.06 \AA$. Measurements were made using silicon cantilever tip (ScienTec AppNano). The cantilever size L: $125 \mu \mathrm{m}$, W: $35 \mu \mathrm{m}$ and T: $4.5 \mu \mathrm{m}$. The tip radius: $<10 \mathrm{~nm}, \mathrm{H}: 14-16 \mu \mathrm{m}$ and with a frequency of $200-400 \mathrm{kHz}$ and spring constant of $\mathrm{K}: 25-75 \mathrm{~N} / \mathrm{m}$. The scanning images were performed at $5 \mathrm{~V}$ amplitude, $8 \mathrm{~V}$ set point and Tip DC at $477 \mathrm{nV}$. Measurements were performed in taping mode with a speed of 0.5 lines per second and 1024 resolution. Samples were analyzed in $\mathrm{a}(5 \mu \mathrm{m} \times 5 \mu \mathrm{m})$ area. 


\subsection{Mott-Schottky analysis}

Mott-Schottky analyses were performed in a conventional $1 \mathrm{~mL}$ electrochemical Teflon cell containing a three-electrode system (Fig. 3). Measurements were made by a VMP3 potentiostat (Biologic-Science Instrumentation, France). Platinum (Pt) and Calomel saturated electrode (CSE) were used as counter and reference electrodes, respectively. Silicon nitride electrodes functionalized with monoclonal anti-OTA antibodies acted as working electrodes. The substrate was sandwiched between the two parts of Teflon cell. Electrical contact was realized through the aluminum layer from the back side of the substrate (Fig. 3). MottSchottky plots were obtained on the films by sweeping the potential from $-1 \mathrm{~V} / \mathrm{SCE}$ to $+3 \mathrm{~V} / \mathrm{SCE}$; at frequency range $2 \mathrm{kHz}-70 \mathrm{kHz}$ and a step rate of $25 \mathrm{mV}$.
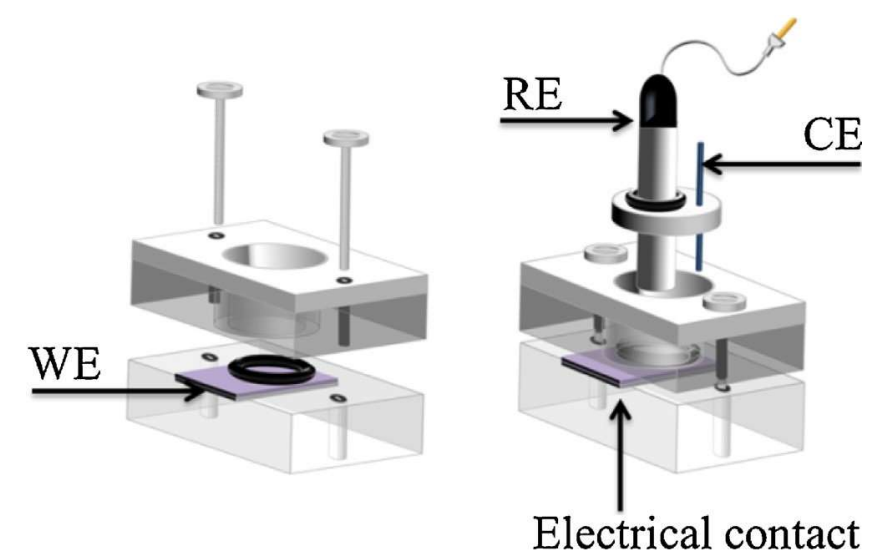

Fig. 3. Teflon electrochemical cell used for electrochemical analysis, with external reference electrode (RE), counter electrode (CE). The working electrode (WE) was sandwiched between the two parts of Teflon cell.

The measurements were carried out in the absence of a redox probe, in PBS at room temperature. The $\mathrm{pH}$ was kept constant throughout all the measurements ( $\mathrm{pH}$ 7.4). All connections were made with coaxial cables to minimize any external electrical noise and the measurements were performed in the dark inside a Faraday cage.

\subsection{Antibodies immobilization}

Silicon nitride substrates were cleaned by sonication in acetone for $15 \mathrm{~min}, 3$ times and washed with distillated water in order to remove the resin protecting layer. Surface activation of the $\mathrm{Si}_{3} \mathrm{~N}_{4}$ was performed using a Piranha solution $\left(1: 3, \mathrm{v} / \mathrm{v}, \mathrm{H}_{2} \mathrm{O}_{2}: \mathrm{H}_{2} \mathrm{SO}_{4}\right)$ for $30 \mathrm{~min}$, and sequential surface chemical treatments in aqueous solutions of $\mathrm{NaOH}(0.5 \mathrm{M})$ for $20 \mathrm{~min}, \mathrm{HCl}(0.1 \mathrm{M})$ for $10 \mathrm{~min}$ and a final immersion in $\mathrm{NaOH}(0.5 \mathrm{M})$ solution for $10 \mathrm{~min}$ [22]. For all these surface chemical treatments, the substrate was floating on the surface and not totally immersed in aqueous solutions. Indeed, silicon nitride was floating and in contact with the solutions leaving the aluminum back side in the air. This procedure was made to protect aluminum layer from all these chemical solutions. Afterward, the samples were then rinsed thoroughly with $\mathrm{HCl}(0.1 \mathrm{M})$ and Milli-Q water and dried in an oven at $120{ }^{\circ} \mathrm{C}$ for $10 \mathrm{~min}$ [22]. 


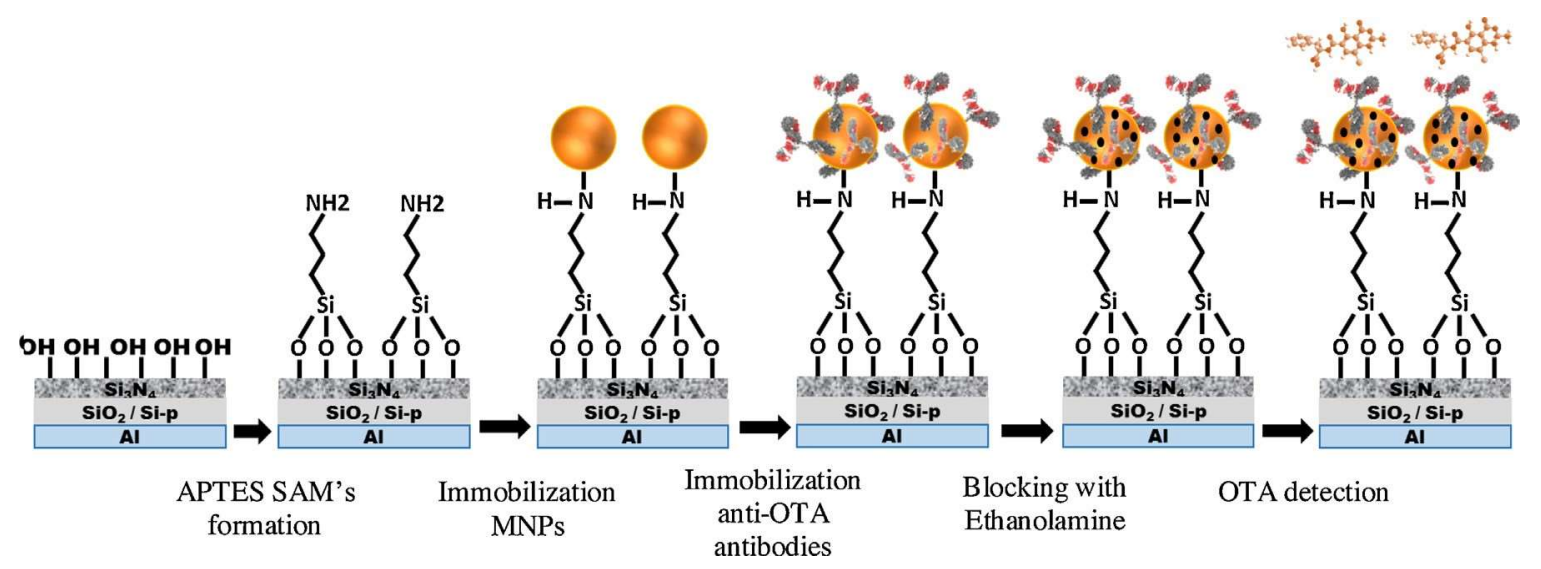

Fig. 4. Schematic representation of the functionalization procedure based on MNPs (๑) and anti-OTA antibodies. Ethanol-amine $(\bullet)$ was used after antibodies immobilization to avoid nonspecific adsorption.
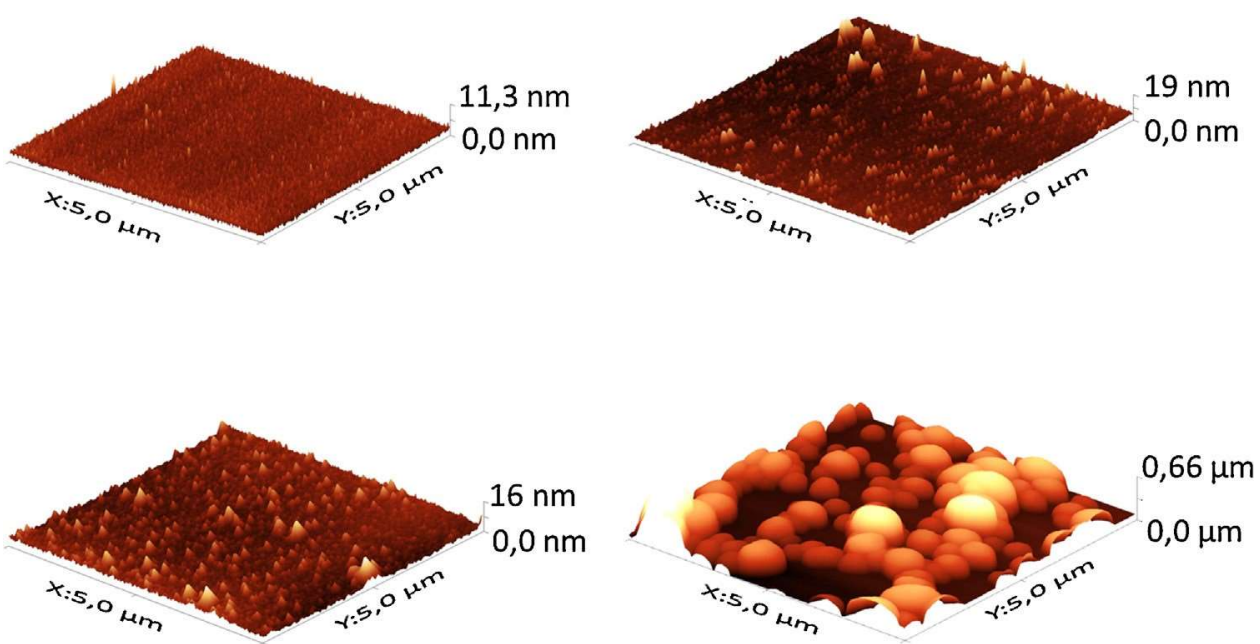

Fig. 5. AFM images of (a) bare (b) Activated, and (c) APTES modified silicon nitride surface. (d) Shows the MNPs distribution onto APTES modified silicon nitride substrate.

Table 1. Contact angle of cleaned substrates, Piranha oxidation, followed by APTES formation on $\mathrm{Si}_{3} \mathrm{~N}_{4}$.

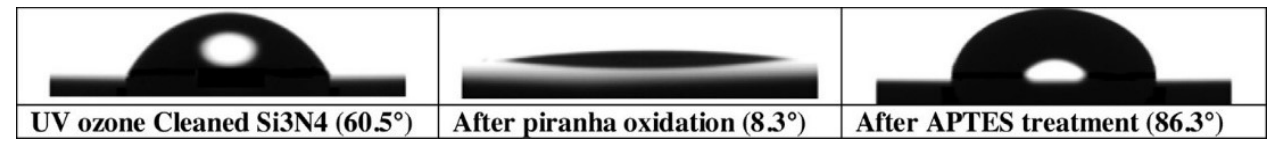

The $\mathrm{Si}_{3} \mathrm{~N}_{4}$ substrates were functionalized using 1\% of APTES in ethanol over night at room temperature. Afterward samples were rinsed with ethanol to remove the unbonded APTES and dried with nitrogen and put in the oven at $100{ }^{\circ} \mathrm{C}$ for one additional hour. Consequently, SAMs of APTES were formed onto the $\mathrm{Si}_{3} \mathrm{~N}_{4}$ surface with amine terminal groups outward from the surface. These were covalently bonded to carboxylic acid groups of the MNPs which have been previously activated with a mixture of EDC (0.4 M), NHS (0.1 M) and $\mathrm{KCl}(1 \mathrm{mM})$ in $500 \mu \mathrm{L}$ MNPs for $3 \mathrm{~h}$ at $37{ }^{\circ} \mathrm{C}$ [26]. This activation enabled the bonding of amine group of APTES with MNPs. Afterwards the immobilized MNPs were rinsed with $\mathrm{HCl}(0.1 \mathrm{M})$ and incubated in anti-OTA solution $(25 \mu \mathrm{g} / \mathrm{mL})$ for $3 \mathrm{hr}$ at room temperature. The monoclonal anti-OTA antibodies were covalently bonded to MNPs though the acid-amine linkage. The residual activated carboxylic acid groups of MNPs were blocked with $1 \%$ of ethanolamine diluted in PBS for $30 \mathrm{~min}$ at room temperature. This step is very 
important to reduce non-specific binding during the detection process. Finally, the biosensor was rinsed with PBS and used for ochratoxin A detection. Fig. 4 depicts the biofunctionalization of the biosensor as detailed in Section 2.

\section{Results and discussion}

\subsection{Contact angle measurements}

In order to assess the effectiveness of the functionalization, contact angle measurements were performed on bare $\mathrm{Si}_{3} \mathrm{~N}_{4}$, before and after activation, and also after its functionalization with APTES. The results in Table 1, demonstrate a slightly hydrophilic nature on bare silicon nitride with a contact angle of $60.5^{\circ}$, which is in agreement with values found in literature [27]. After surface oxidation with Piranha, $\mathrm{Si}_{3} \mathrm{~N}_{4}$ surface becomes highly hydrophilic $8.3^{\circ}$. This was due to the presence of a high amount of hydroxyl groups on the surface. The contact angle has increased again to $86.3^{\circ}$ after the functionalization process with the APTES. This hydrophobic character can be explained by the presence of APTES hydrocarbon chains.
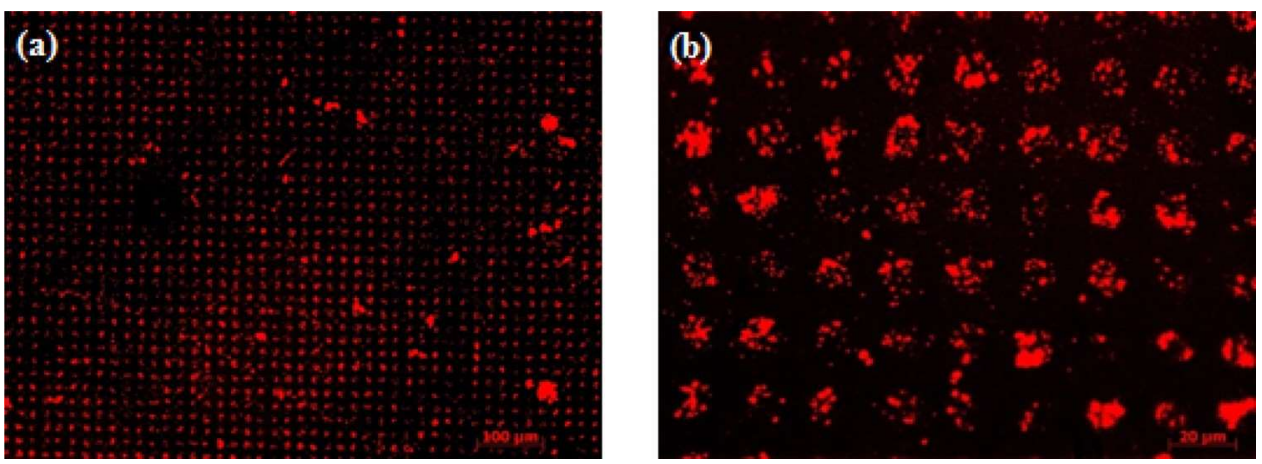

Fig.6. Fluorescent image of (a) a homogenous pattern of MNP/anti-OTA antibodies after recognition of the corresponding antigen (b) magnification of the positive pattern showing specific detection by the immobilized anti-OTA.

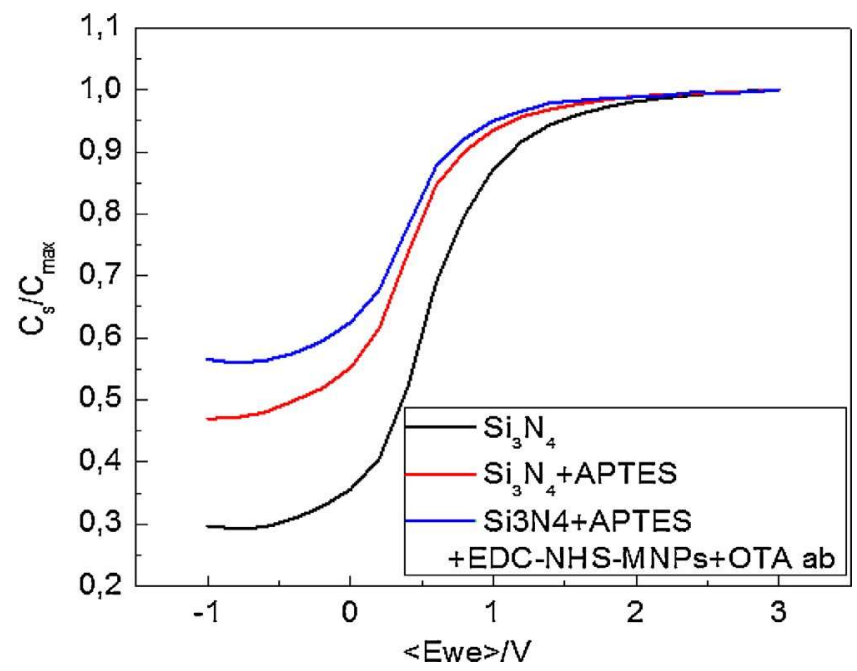

Fig. 7. Mott-Schottky plots of the electrodes: (a) $\mathrm{Si}_{3} \mathrm{~N}_{4}$, (b) $\mathrm{Si}_{3} \mathrm{~N}_{4}+$ APTES, (c) $\mathrm{Si}_{3} \mathrm{~N}_{4}+$ APTES + EDC-NHS$\mathrm{MNP}+\mathrm{Ab}-\mathrm{OTA}$. 


\subsection{Atomic force and scanning electron microscopy}

Fig. 5 shows the topography of the silicon nitride modified surfaces, observed by AFM compared to bare $\mathrm{Si}_{3} \mathrm{~N}_{4}$ electrode (Fig. 5a). The roughness of the activated $\mathrm{Si}_{3} \mathrm{~N}_{4}(0.337 \mathrm{~nm})$ was clearly higher than the bare $\mathrm{Si}_{3} \mathrm{~N}_{4}$ surface $(0.152 \mathrm{~nm})$ which confirm the good activation of the surface (Fig. 5b). After the passivation of $\mathrm{Si}_{3} \mathrm{~N}_{4}$ with APTES, the roughness was changed from $0.337 \mathrm{~nm}$ to $0.23 \mathrm{~nm}$ indicating the good formation of APTES SAMs onto the electrode surface (Fig. 5c). Finally, the AFM image of the MNPs shows good coverage of the surface of the electrode with homogeneous distribution (see Fig. 5d). The MNPs contribute to the high roughness on the modified silicon nitride surface. The layer of MNPs presents a very high textured surface with average depth of $\sim 0.2 \mu \mathrm{m}$ and average roughness of $13.5 \mathrm{~nm}$. This configuration is very important for 3D bio-functionalization (Fig. S2 Supplementary material). This configuration allows the immobilization of a large amount of antibodies which can increase the sensitivity of the biosensor. Fig. S3 in Supplementary material shows a SEM image of MNPs onto silicon substrate and confirms the homogenous distribution of MNPs.

\subsection{Fluorescence analysis}

Fluorescent imaging is a rapid tool for analyzing bio-layers. This technique is based on the reaction of labeled fluorochrome biomolecules with the corresponding bioreceptor in order to ensure detection or bio-recognition processes. In the present study OTA is on itself fluorescent, and it has an emission maximum at $467 \mathrm{~nm}$ in $96 \%$ ethanol and $428 \mathrm{~nm}$ in absolute ethanol after excitation at $340 \mathrm{~nm}$ [28]. The soft-lithographical technique, $\mu \mathrm{CP}$, facilitates the printing of the required pattern by applying a structured PDMS stamp as mentioned in previous paragraph. Fig. 6 shows fluorescent pattern of OTA. The labeled fluorescent tags formulate positive structures, while the non-fluorescent regions were blocked with OTS. Here well-proportioned positive patterns were shown with perfectly immobilized OTA antigens.

\subsection{Mott-Schottky results}

Mott-Schottky analyses were performed in order to characterize the semi-conducting behavior of silicon nitride substrate after each step of chemical surface modification (SAMs of APTES and the MNPs/anti-OTA antibodies). Fig. 7 shows the Mott-Schottky plots evolution for bare silicon nitride, APTES modified silicon nitride and after MNPs/anti-OTA antibodies immobilization at the frequency of $70 \mathrm{kHz}$. This latter has been optimized and chosen as the appropriate frequency, as it gives a better capacitive behavior (Fig. S4 Supplementary material). The capacitance of the substrate $\left(\mathrm{C}_{\mathrm{s}}\right)$ was normalized by dividing the value of $\mathrm{C}_{\mathrm{s}}$ by the maximum value of each curve of $C(V)$ and presented as $C_{s} / C_{\max }$ versus the potential of the working electrode $\left(\mathrm{E}_{\mathrm{WE}}\right)$. Here, the capacitance of the APTES modified silicon nitride has increased when compared to the bare $\mathrm{Si}_{3} \mathrm{~N}_{4}$ due to the formation of new layer onto $\mathrm{Si}_{3} \mathrm{~N}_{4}$ surface. The same behavior of the capacitance increase has been observed after MNPs/anti-OTA antibodies immobilization onto APTES monolayer.

After MNPs/anti-OTA antibodies immobilization, Mott-Schottky analysis has been performed in order to determine blank measurements (Fig. 8a). Then the biosensor was maintained in the electrochemical cell and incubated for $30 \mathrm{~min}$ in $1 \mathrm{~mL}$ of PBS containing OTA antigens at $4{ }^{\circ} \mathrm{C}$. The biosensor was then rinsed with PBS in order to remove any adsorbed proteins and analyzed afterward by Mott-Schottky using $1 \mathrm{~mL}$ of PBS as electrolyte. This procedure of the biosensor incubation was made for all OTA concentrations. The detection of OTA antigens at various concentrations is shown in Fig. $8 \mathrm{a}$. Here the $\mathrm{C}_{\mathrm{s}} / \mathrm{C}_{\mathrm{max}}$ shows a shift in the X-direction by increasing OTA concentrations, which confirms a built-in potential difference, equivalent to a flat band voltage variation, and thus an increase in the conduction current value. Based on all these elements, the biosensor sensitivity can be obtained by measuring the potential shift of the flat bond of the $C_{s}=f(V)$ curves. 

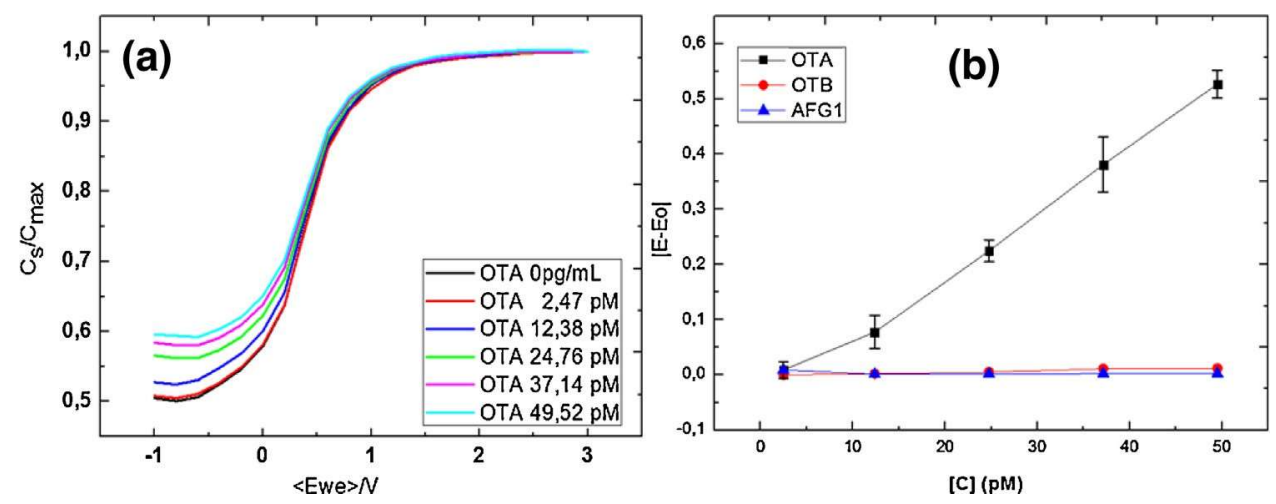

Fig. 8. (a) Mott-Schottky plots for OTA detection using the capacitance biosensor, (b) The Calibration curves of the OTA detection (black curve), and of the two interferences: OTB (red curve) and AFG1 (blue curve). (For interpretation of the references to colour in this figure legend, the reader is referred to the web version of this article.)
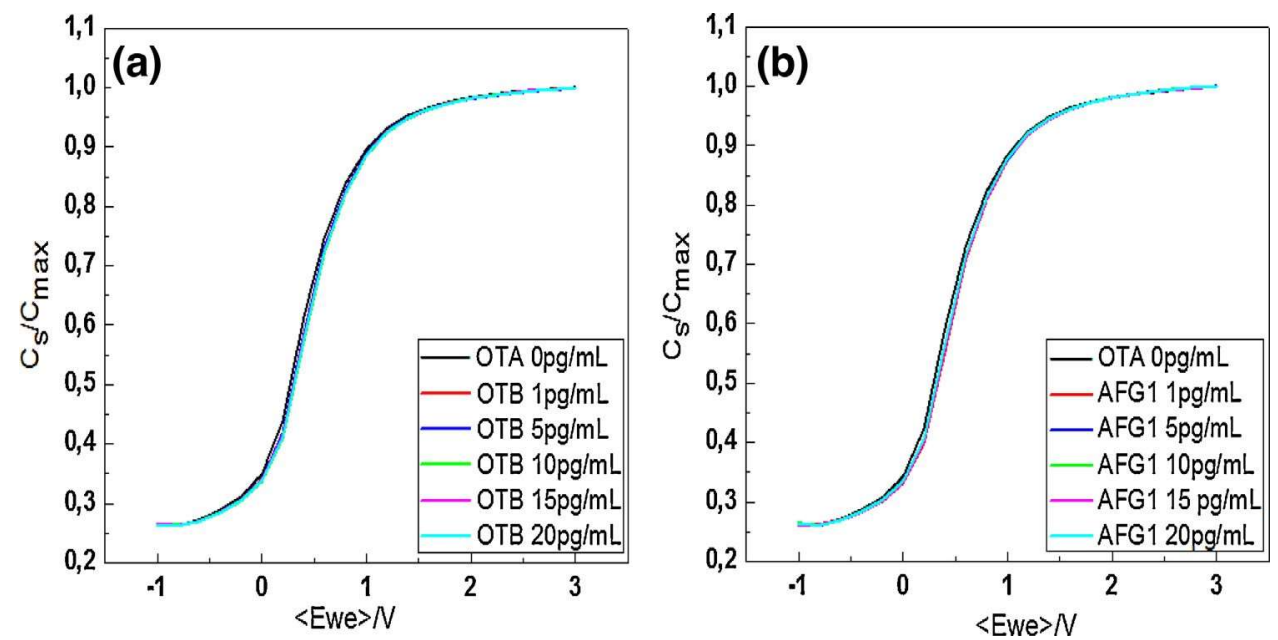

Fig. 9. (a) Mott-Schottky plots for OTB detection and (b) for AFG1 detection using the capacitance biosensor.

Fig. 8b shows the calibration curve of the capacitive biosensor based on silicon nitride in a linear range of $2.47-49.52 \mathrm{pM}$ of OTA. Calibration curve was presented as the absolute value of relative variation of potential $\mid \mathrm{E}-\mathrm{E}$ | versus concentration of OTA in pM, where $\mathrm{E}$ is the potential of different OTA concentrations and Eo is the potential of the antibodies anti-OTA. The developed biosensor provides a high sensitivity of $0.01 \mathrm{VpM}^{-1}$, and a good linearity with a correlation coefficient $\left(\mathrm{R}^{2}\right)$ of 0.99 . The limit of detection of this capacitive biosensor was defined at $4.57 \mathrm{pM}$.

The specificity of the prepared biosensor was realized using ochratoxin B (OTB) and aflatoxin G1 (AFG1) other compounds with similar structures. Mott-Schottky analyses were carried out, using the same experimental process based on MNPs/anti-OTA antibodies immobilization. The detection of OTB and AFG1 was made in the same linear range of 2.47-49.52 pM. The potential difference of both OTB and AFG1 was negligible when compared to OTA detection Fig. 9. This shows an excellent specificity and sensitivity of the biosensor for OTA antigens (Fig. 8b) and can offer credible results despite the presence of the interfering species. 


\section{Conclusion}

Novel sensitive and selective biosensor based on $\mathrm{Si}-\mathrm{p} / \mathrm{SiO}_{2} / \mathrm{Si}_{3} \mathrm{~N}_{4}$ structure combined with new mangnetic nanoparticles for the specific detection of OTA was developed. $\mathrm{Si}_{3} \mathrm{~N}_{4}$ was chosen for its many attractive physical and chemical properties as well as its stability for long periods. Moreover, making electrochemical analysis using this material provides a stable measurement and rapid response.

Under optimized conditions, the developed biosensor provides a high sensitivity of $0.01 \mathrm{VpM}-1$, and a limit of detection of $4.57 \mathrm{pM}$ was obtained. The biosensor was highly selective for ochratoxin A antigens, when compared to other interferences ochratoxin B and aflatoxin G1. The proposed method is very promising for OTA detection for several agro-food industry applications. To our knowledge this is the first silicon nitride biosensor reported for the detection of OTA.

\section{Acknowledgments}

We acknowledge the funding through the SMARTCANCERSENS project (FP7-PEOPLE-2012-IRSES) under the grant agreement No. 31805, the SEA-on-a-CHIP project (FP7-KBBE) under the reference 614168, the NATO project, SPS (NUKP.SFPP984173) and the HEARTEN under the grant agreement No. 643694.

\section{Appendix A. Supplementary data}

Supplementary data associated with this article can be found, in the online version, at http://dx.doi.org/10.1016/j.snb.2016.03.166.

\section{References}

[1] A. Jodra, M. Hervás, M. ÁngelLópez, A. Escarpa, Disposable electrochemical magneto immunosensor for simultaneous simplified calibration and determination of Ochratoxin A in coffee samples, Sens. Actuators B 221 (2015) 777-783.

[2] F. Gentile, G.L. La Torre, A.G. Potortì, M. Saitta, M. Alfa, G. Dugo, Organic wine safety: UPLC-FLD determination of Ochratoxin A in Southern Italy wines from organic farming and winemaking, Food Control 59 (2016) 20-26.

[3] F. Dridi, M. Marrakchi, M. Gargouri, A. Garcia-Cruz, S. Dzyadevych, F. Vocanson, J. Saulnier, N. Jaffrezic-Renault, F. Lagarde, Thermolysin entrapped in a gold nanoparticles/polymer composite for direct and sensitive conductometric biosensing of ochratoxin A in olive oil, Sens. Actuators B 221 (2015) 480-490.

[4] European Commission, Commission regulation 472/2002 of 12 March 2002 amending regulation (EC) N. 466/2001 setting maximum levels for certain contaminants in foodstuffs, Off. J. Eur. Commun. L75 (2002) 18-20.

[5] J. Mao, S. Lei, X. Yang, D. Xiao, Quantification of ochratoxin A in red wines by conventional HPLCFLD using a column packed with core-shell particles, Food Control 32 (2013) 505-511.

[6] S. Kirin, B. Skrjanc, N. Kos, B. Kozolc, N. Pirnat, G. Tavcar-Kalcher, Mycotoxins in cereals and cereal products in Slovenia - official control of foods in the years 2008-2012, Food Control 50 (2015) 157165. 
[7] A. Zhang, Y. Ma, L. Feng, Y. Wang, C. He, X. Wang, H. Zhang, Development of a sensitive competitive indirect ELISA method for determination of ochratoxin A levels in cereals originating from Nanjing, China, Food Control 22 (2011) 1723-1728.

[8] B. Prieto-Simon, M. Campàs, J. Marty, T. Noguer, Novel highly-performing immunosensor-based strategy for ochratoxin A detection in wine samples, Biosens. Bioelectron. 23 (2008) 995-1002.

[9] X.P. Liu, Y.J. Deng, X.Y. Jin, L.G. Chen, J.H. Jiang, G.L. Shen, R.Q. Yu,

[10] Ultrasensitive electrochemical immunosensor for ochratoxin A using gold colloid-mediated hapten immobilization, Anal. Biochem. 389 (2009) 63-68.

[11] S.H. Alarc on, G. Palleschi, D. Compagnone, M. Pascale, A. Visconti, I. Barna-Vetro, Monoclonal antibody based electrochemical immunosensor for the determination of ochratoxin $\mathrm{A}$ in wheat, Talanta 69 (2006) 1031-1037.

[12] M.M. Barsan, M.E. Ghica, C.M.A. Brett, Electrochemical sensors and biosensors based on redox polymer/carbon nanotube modified electrodes: a review, Anal. Chim. Acta (2015) (accepted manuscript).

[13] K. Awsiuk, A. Bernasik, M. Kitsara, A. Budkowski, P. Petrou, S. Kakabakos, S. Prauzner-Bechcicki, J. Rysz, I. Raptis, Spectroscopic and microscopic characterization of biosensor surfaces with protein/amino-organosilane/silicon structure, Colloids Surf. B 90 (2012) 159-168.

[14] N.H.Z. Ariffin, H. Yahaya, S. Shinano, S. Tanaka, A.M. Hashim, Fabrication of conical micropore structure on silicon nitride/silicon using focused ion beam milling for biosensor application, Microelectron. Eng. 133 (2015) 1-5.

[15] Q. Liu, X. Tu, K.W. Kim, J.S. Kee, Y. Shin, K. Han, Y.-J. Yoon, G.-Q. Lo, M.K. Park, Highly sensitive Mach-Zehnder interferometer biosensor based on silicon nitride slot waveguide, Sens. Actuators B 188 (2013) 681-688.

[16] P.M. Levinea, P. Gonga, R. Levickyb, K.L. Sheparda, Real-time, multiplexed electrochemical DNA detection using an active complementary metal-oxide-semiconductor biosensor array with integrated sensor electronics, Biosens. Bioelectron. 24 (2009) 1995-2001.

[17] J.Y. Kim, K. Choi, D. Moon, J.H. Ahn, T.J. Park, S.Y. Lee, Y.K. Choi, Surface engineering for enhancement of sensitivity in an underlap-FET biosensor by control of wettability, Biosens. Bioelectron. 41 (2013) 867-870.

[18] A. Szwajca, J. Wei, M.I. Schukfeh, M. Tornowa, Self-assembled monolayers of alkyl-thiols on InAs: a Kelvin probe force microscopy study, Surf. Sci. 633 (2015) 53-59.

[19] F.C. Fernandes, A. Santos, D.C. Martins, M.S. Góes, P.R. Bueno, Comparing label free electrochemical impedimetric and capacitive biosensing architectures, Biosens. Bioelectron. 57 (2014) 96-102.

[20] M.S. Góes, J.J. Davis, P.R. Bueno, Label free redox capacitive biosensing, Biosens. Bioelectron. 50 (2013) 437-440.

[21] J. Lehr, F.C. Bedatty, P.R. Bueno, J.J. Davis, Label-free capacitive diagnostics: exploiting local redox probe state occupancy, Anal. Chem. 86 (2014) 2559-2564.

[22] K. Awsiuk, A. Bernasik, M. Kitsara, A. Budkowski, J. Rysz, J. Haberko, P. Petrou, K. Beltsios, J. Raczkowska, Protein coverage on silicon surfaces modified with amino-organic films: a study by AFM and angle-resolved XPS, Colloids Surf. B 80 (2010) 63-71.

[23] D. Caballero, J. Samitier, J. Bausells, A. Errachid, Direct patterning of anti-Human serum albumin antibodies on aldehyde-Terminated silicon nitride surfaces for HSA protein detection, Small 5 (13) (2009) 1531-1534.

[24] C. Jianrong, M. Yuqing, H. Nongyue, W. Xiaohue, L. Sijiao, Nanotechnology and biosensors, Biotechnol. Adv. 22 (2004) 505-518. 
[25] W.M. Hassen, L. Angnes, A. Abdelghani, F. Bessueille, D. Leonard, N. Jaffrezic-Renault, Under flow impedimetric measurements using magnetic particles for label-free detection affinity target, Mater. Scie. Eng. C 28 (2008) 820-825.

[26] R. Rawal, S. Chawla, C.S. Pundir, An electrochemical sulfite biosensor based on gold coated magnetic nanoparticles modified gold electrode, Biosens.

[27] Bioelectron. 31 (2012) 144-150.

[28] L.G. Zamfir, I. Geana, S. Bourigua, L. Rotariua, C. Bala, A. Errachid, N.

[29] Jaffrezic-Renault, Highly sensitive label-free immunosensor for ochratoxin A based on functionalized magnetic nanoparticles and EIS/SPR detection, Sens.

[30] Actuators B 159 (2011) 178-184.

[31] D. Caballero, E. Martinez, J. Bausells, A. Errachid, J. Samitier, Impedimetric immunosensor for human serum albumin detection on a direct aldehyde-functionalized silicon nitride surface, Anal. Chim. Acta 720 (2012) 43-48.

[32] K. Biró, L. Solti, I. Barna-Vetró, G. Bagó, R. Glávits, E. Szabó, J. Fink-Gremmels, Tissue distribution of ochratoxin A as determined by HPLC and ELISA and histopathological effects in chickens, Avian Pathol. 31 (2) (2010) 141-148.

\section{Biographies}

Madiha Bougrini is currently a PhD student in the Institute of Analytical Sciences at the University of Lyon and in the Laboratory of Electronics, Automatic and Biotechnology, University Moulay Ismail, Faculty of Sciences in Meknes, Morocco. Her research interests include sensors networks based electronic noses/tongues for food analysis and the design of electrochemical biosensors.

Abdoullatif Baraket received his B.S. in physics and M.S. in scientific computing in physics and experimental high energy from Hassan 2 University in 2006 and 2008 respectively. He also received a M.S. in physics and nanomaterial from the University of Maine in 2009. He obtained his Ph.D at the Université de Lyon in 2013 and afterwards he was a postdoctoral researcher at the Commissariat à l'énergie atomique (CEA) Grenoble. He is now a postdoctoral researcher in the group of Prof. Abdelhamid Errachid.

Joan Bausells was born in Barcelona, Spain, in 1957. He graduated in physics in 1980, and received M.S. (1982) and Ph.D. (1986) degrees in solid-state physics, all from the University of Barcelona. From 1981 to 1986 he worked as an R\&D engineer in the semiconductor industry. In 1986 he joined IMB-CNM, where he is a permanent researcher since 1988 and Research Professor since 2002. At IMB-CNM he was manager of the Sensor and Actuator Group (1990-1995), the Microsystems Department (1999-2002) and the Nanotechnology Group (2002-2006). From 2008 to 2012 he was Deputy Director of IMB-CNM, and Acting Director from January to June 2012. He has published more than 90 papers in international journals, and has contributed more than 220 papers to Conferences. He has participated in 14 projects funded by the EC Framework Programmes in the micro- and nano-systems field, and has coordinated two of them. His current research interests include micro- and nanoelectromechanical structures and nanoelectronic devices, and their applications to micro/nano (sensor)systems based on silicon.

Miguel Zabala graduated in telecommunications engineering at the Universitat Politècnica de Catalunya, Barcelona, in 1994. In 1995, he joined the Centro Nacional de Microelectronica (CNM) where he is currently working. His main area of interest is thermal process engineering for nano-microelectronic technologies and analog circuitry for microsystems.

Nazha El Bari received her PhD degree in 1989 from the University of Nancy (France). She joined the University of Moulay Ismail in 1990, and she was a wanted a Doctor of Sciences degree in 1995 in this 
university. She has been Professor since 1999, and she created a Biotechnology research group in 2005. Her research interests include quality control of milk and their products.

Benachir Bouchikhi received his PhD degree in 1982 from the University of Aix Marseille (France). He joined the University of Nancy I in the same year, and he award a Doctor of sciences degree in 1988. In 1994, he has been Professor at the Faculty of Sciences of Moulay Ismail University (Morocco), and he created a Sensors, Electronics and Instrumentation Group in 2005. His current major research interests involve electronic nose and characterization of thin film chemical sensors.

Abdelhamid Errachid is a Full Professor in Claude Bernard University-Lyon 1 since the end of 2008. He has been involved as a principal investigator and team leader in several European Projects under 5th, 6th, and 7th Framework Programmes: FP6 (DVT-IMP, MAPTech, Nano2Life, Cell-PROM, ARES, VECTOR, SPOTNOSED) and FP7 (SensorART, BOND, SEA-on-a-CHIP) as well as NATO, INTAS and TEMPUS International Projects, and Spanish national projects (MICROMENCE, MINAHE I, MINAHE II, and PETRI). Prof. Errachid is a head of the SIMS (Surface-(bio) Interfaces-Micro/nano Systems") group from 2010 to 2014. Papers: 130, Congress: 322, h-index: 219, Patents: 3 (1 PTC), Publications: Books: 8. His current research activity is focused in BioElectronics, Biofunctionalization, and NanoBiotechnology.

Nadia Zine received her B.S. in chemistry from the University M. Ismail, Meknes in 1994 and her Ph.D. from the Centro Nacional de Microelectrónica (CNM) Barcelona in 2005. Currently, she is a lecturer/researcher at the Université Claude Bernard Lyon 1. Her research interests are within chemical characterization of silicon chemical sensors, and specially, of ISFET devices. 